\title{
Relative dispersion of a passive scalar plume in turbulent shear flow
}

\author{
Christina Vanderwel and Stavros Tavoularis* \\ Department of Mechanical Engineering, University of Ottawa, Ottawa, Ontario, Canada K1N 6N5
}

(Received 23 February 2014; published 24 April 2014)

\begin{abstract}
Relative dispersion of a passive scalar plume was investigated in uniformly sheared, nearly homogeneous, turbulent flow with $\mathrm{Re}_{\lambda} \approx 150$ using planar laser-induced fluorescence. Mean concentration maps were determined both in the laboratory frame and in a frame attached to the instantaneous center of mass of the plume cross section. The distance-neighbor function had a shape that was compatible with Richardson's expression. The mean square particle separation, two estimates of which were found to be nearly identical, had a streamwise evolution that was consistent with Richardson-Obukhov scaling with a Richardson's constant of $g=0.35$. Batchelor scaling was also consistent with a wide range of the results.
\end{abstract}

DOI: 10.1103/PhysRevE.89.041005

PACS number(s): 47.27.tb, 47.27.Gs

Relative dispersion is concerned with the temporal evolution of the separation distance $r(t)$ of a pair of fluid particles in turbulent flows. The concept of relative dispersion was introduced by Richardson [1], who in 1926 proposed his famed four-thirds law. In this Rapid Communication, we focus on relative dispersion in the inertial subrange of turbulent motions, namely, for $\eta \ll r \ll L_{11,1}$, where $\eta$ is the Kolmogorov microscale and $L_{11,1}$ is the integral length scale of the turbulence. Within this range, relative dispersion would be dominated by eddies with sizes comparable to $r$, and the variance $\left\langle r^{2}(t)\right\rangle$ of separations of a large number of particle pairs can be estimated as [2]

$$
\left\langle r^{2}(t)\right\rangle-r_{0}^{2}= \begin{cases}\frac{11}{3} C_{2}\left(\langle\epsilon\rangle r_{0}\right)^{2 / 3} t^{2} & \text { for } t \ll t_{B} \\ g\langle\epsilon\rangle t^{3} & \text { for } t_{B} \ll t \ll T_{L} .\end{cases}
$$

In these expressions, $r_{0}$ is the initial pair separation, $C_{2}$ is the Kolmogorov constant [3,4], $\langle\epsilon\rangle$ is the mean dissipation rate, $t_{B}=\left(r_{0}^{2} /\langle\epsilon\rangle\right)^{1 / 3}$ is the Batchelor time scale, $g$ is the Richardson constant, and $T_{L}$ is the Lagrangian integral time scale. The range $t \ll t_{B}$ is known as the Batchelor regime, whereas the term Richardson-Obukhov regime signifies the range $t_{B} \ll t \ll T_{L}$. Theoretical estimates of Richardson's constant span the wide range $0.06<g<3.52$ [5], whereas recent experimental and numerical estimates have narrowed it down to the range $0.5<g<0.6$ [2].

Particle separations have been measured directly with the use of particle tracking methods [2,4,6-8]. Alternatively, mean particle separation can also be estimated from measurements of concentration in a diffusing cloud of particles relative to its center of mass [5,9-11]. A property of interest in such studies is the distance-neighbor function $q(s)$, defined as the probability density function (pdf) of the distance $s$ between particle pairs within the cloud [1,5]. $q(s)$ can be estimated as the marginal pdf of the ensemble-averaged autocorrelations of instantaneous concentration maps [12]. In the Richardson-Obukhov regime, theoretical predictions have led to the relationship $q(s) \propto$ $e^{-s^{n}}$; the exponent $n$ was given as $2 / 3$ by Richardson and as $n=2$ by Batchelor [2], but values of $n$ from particle tracking measurements [6-8] and concentration maps [12] have so far been inconsistent. The accuracy of relative diffusion

\footnotetext{
*stavros.tavoularis@uottawa.ca
}

measurements in laboratory studies has improved significantly in recent years, following advances in planar laser-induced fluorescence and particle tracking techniques. Even so, the fact that most laboratory flows have been conducted at relatively low turbulence Reynolds numbers $\mathrm{Re}_{\lambda}$ limits their assertiveness, particularly in the light of the ongoing debate concerning the existence of the Richardson-Obukhov regime at low Reynolds numbers. Bourgoin et al. [4] studied the motion of particles in a closed tank stirred by counter-rotating baffled disks and observed that the evolution of $\left\langle r^{2}(t)\right\rangle$ was consistent with Batchelor scaling for $t \ll t_{B}$, but found no evidence of Richardson-Obukhov scaling for $t \gg t_{B}$, for values of the turbulence Reynolds number $\mathrm{Re}_{\lambda}$ up to 815 . In contrast, Ott and Mann [7] reported evidence of Richardson-Obukhov scaling in a turbulent flow generated in a closed tank by oscillating grids at $\operatorname{Re}_{\lambda} \sim 100$.

The general objective of the present research was to investigate the relative dispersion of a passive scalar plume in nearly homogeneous but strongly anisotropic turbulence with a moderate Reynolds number. Specific objectives were to determine whether Richardson-Obukhov scaling applies to this flow and to estimate the shape of the distance-neighbor function and the rate of growth of the mean particle separation.

Experiments were performed in nearly homogeneous, uniformly sheared flow (USF), in a free-surface, recirculating water tunnel (see Fig. 1). A uniform velocity gradient in the $x_{2}$ direction was generated by a shear generator, which consisted of an array of parallel channels with a spacing $L=25.4 \mathrm{~mm}$ and varying flow obstruction. A neutrally buoyant aqueous solution of rhodamine $6 \mathrm{G}$ fluorescent dye was injected isokinetically into the flow through a fine tube having an inner diameter of $1.83 \mathrm{~mm}$.

The turbulence structure of the USF has been documented previously [13,14]; representative values of properties of interest are presented in Table I. The initial mean particle separation, determined as the second central moment of the concentration profile at the outlet of the injection tube, was $r_{0}=0.53 \mathrm{~mm}$, which corresponded to a Batchelor time scale $t_{B}=\left(r_{0}^{2} /\langle\epsilon\rangle\right)^{1 / 3} \approx 0.14 \mathrm{~s}$. As explained in a previous article [14], the streamwise integral Lagrangian time scale was estimated as $T_{L} \approx 2.6 L_{22,2} / u_{2}^{\prime} \approx 6.1 \mathrm{~s}$. Based on these values, one would expect the Richardson-Obukhov regime to extend over the diffusion time range $0.14 \mathrm{~s} \ll t \ll 6.1 \mathrm{~s}$; 


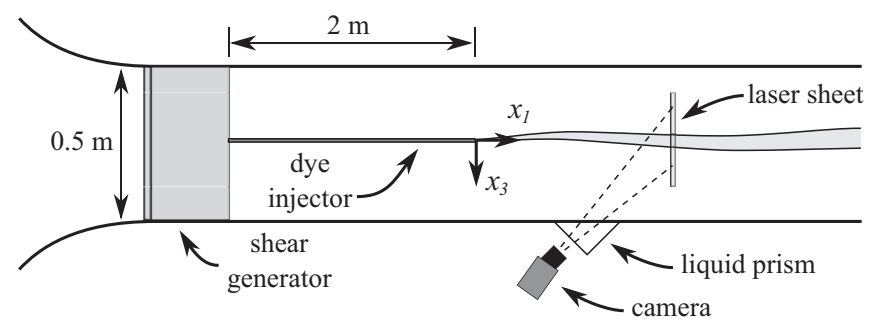

FIG. 1. Sketch of the apparatus viewed from above; the $x_{2}$ axis is oriented out of the page.

further employing Taylor's frozen flow approximation as $t \approx x_{1} / U_{C}$, one may estimate that the Richardson-Obhukhov regime would extend over the range of streamwise distances $1 \ll x_{1} / L \ll 45$, which applies to all presently reported measurements. Measurements of the concentration in cross sections of the plume normal to the flow direction were obtained via planar laser-induced fluorescence at $x_{1} / L=$ $5,12,20,28$, and 35. Normal cross sections were chosen in preference to the streamwise cross sections used in other studies [12], which cannot track the plume when it wanders out of the plane. Plume cross sections were illuminated by a light sheet created from the output of a Nd:YAG pulsed laser (New Wave Research, Solo PIV 120XT, Fremont, CA, USA) and the resulting fluorescence was recorded using a digital camera (PCO AG, pco.edge, Kelheim, Germany) which was synchronized with the laser pulses. The camera was fitted with a Scheimpflug adapter, in order to focus on the oblique measurement plane, and a liquid prism was used to eliminate the horizontal astigmatism that would have been introduced from looking through the glass wall at an angle. The concentration $C$ of dye measured by each pixel of the camera was determined as a linear function of the local radiant intensity emitted by the dye in the plane of the laser sheet. Ensemble-averaged quantities were calculated based on typically 500 independent images at each location. Additional details on the PLIF technique and its uncertainty will be the subject of a future manuscript. In summary, the uncertainty of the reported values of the plume widths and other length scales was lower than $5 \%$.

A representative instantaneous concentration map is presented in Fig. 2(a). At this particular instant, the entire plume was located off-center and towards the top and right side of the

TABLE I. Representative values of flow parameters.

Shear generator spacing, $L=25.4 \mathrm{~mm}$

Mean center line velocity, $U_{C}=0.18 \mathrm{~m} / \mathrm{s}$

Mean velocity gradient, $d \bar{U}_{1} / d x_{2}=0.59 \mathrm{~s}^{-1}$

Turbulence Reynolds number, $\operatorname{Re}_{\lambda}=150$

Turbulent kinetic energy dissipation rate, $\langle\epsilon\rangle=7.6 \mathrm{~mm}^{2} \mathrm{~s}^{-3}$

Kolmogorov microscale, $\eta=0.6 \mathrm{~mm}$

Taylor microscale, $\lambda_{11}=17 \mathrm{~mm}$

Eulerian integral length scale, $L_{11,1}=30 \mathrm{~mm}$

Lagrangian integral time scale, $T_{L}=6.1 \mathrm{~s}$

Dye Schmidt number, $\mathrm{Sc}=2500$

Dye source concentration, $C_{S}=0.3 \mathrm{mg} / \ell$ field of view; such relatively large displacements are identified as plume wandering and are attributed to turbulent eddies with scales larger than the plume width. The plume cross section was not simply connected, but consisted of multiple elongated filaments; this action is attributed to eddies with size comparable to the plume width. Moreover, the edges of the filaments were not rounded, but showed evidence of the action of eddies with sizes much smaller than the plume width. The center of mass of the instantaneous concentration map in Fig. 2(a) is marked with a cross. Its coordinates $x_{2 C}$ and $x_{3 C}$ were determined from the corresponding first moments of the concentration map.

The map of the mean concentration $\left\langle C\left(x_{2}, x_{3}\right)\right\rangle$ in the laboratory frame is plotted in Fig. 2(b). It can be seen that the mean map extended over a much larger area than the instantaneous one, as the result of large-scale plume wandering. The joint probability density function (jpdf) of the center of mass coordinates in the instantaneous maps is presented in Fig. 2(c). A coordinate transformation of each instantaneous map to a frame centered on the instantaneous center of mass resulted in instantaneous concentration maps in a wandering frame. The ensemble average $C_{R}\left(x_{2}, x_{3}\right)=$ $\left\langle C\left(x_{2}-x_{2 C}, x_{3}-x_{3 C}\right)\right\rangle$ of these maps, to be referred to as the "relative" concentration map [15], is shown in Fig. 2(d). The absolute mean concentration map, the relative mean concentration map, and the jpdf of the instantaneous centers of mass could all be approximated by two-dimensional (2D) Gaussian functions, contours of which are superimposed on the maps in Fig. 2.

The half-widths of each map in the directions $x_{2}$ and $x_{3}$ were defined as the square roots of the corresponding second central moments. Following these definitions, we calculated the half-widths $\sigma_{2}, \sigma_{3}$ of the mean concentration map, $\sigma_{R 2}, \sigma_{R 3}$ of the relative mean concentration map, and $\sigma_{M 2}, \sigma_{M 3}$ of the jpdf of the instantaneous centers of mass. It is noted that $\sigma_{3} / \sigma_{2} \approx \sigma_{M 3} / \sigma_{M 2} \approx 1.25$, which is consistent with the Reynolds stress anisotropy in USF [13]. In contrast, $\sigma_{R 3} / \sigma_{R 2} \approx 1$.1, which is closer to unity, in conformity with the conjecture that relative dispersion is dominated by eddies in the inertial subrange, which would be locally isotropic. The streamwise evolutions of the three mean half-widths $\sigma, \sigma_{R}$, and $\sigma_{M}$, computed by averaging the corresponding half-widths in the two directions, are presented in Fig. 3. The three values satisfy the relationship $\sigma^{2}=\sigma_{R}^{2}+\sigma_{M}^{2}$, in conformity with the parallel axis theorem of moments of inertia. The trends of the half-widths are in excellent agreement with the idealized trends presented by Csanady [15, Fig. 4.4a]. Within the range of reported measurements, the scale of meandering $\sigma_{M}$ was comparable to the relative plume width $\sigma_{R}$, in support of the conjecture that dye dispersion was dominated by eddies comparable in size to the width of the instantaneous plume. In the range $x_{1} / L \leqslant 20$, all three widths grew approximately linearly, whereas in the following range, having $20<x_{1} / L \leqslant$ 37, $\sigma$ and $\sigma_{R}$ maintained their quasilinear growth but the growth rate of $\sigma_{M}$ decreased monotonically, possibly tending towards a zero asymptote; the latter observation agrees with the expectation that, in the far field of the plume, $\sigma_{M}$ would approach an asymptotic value of the order of $L_{11,1}$ [15].

Next, we estimated the distance-neighbor functions $q\left(s_{2}\right)$ and $q\left(s_{3}\right)$ as the marginal pdfs of the ensemble-averaged 

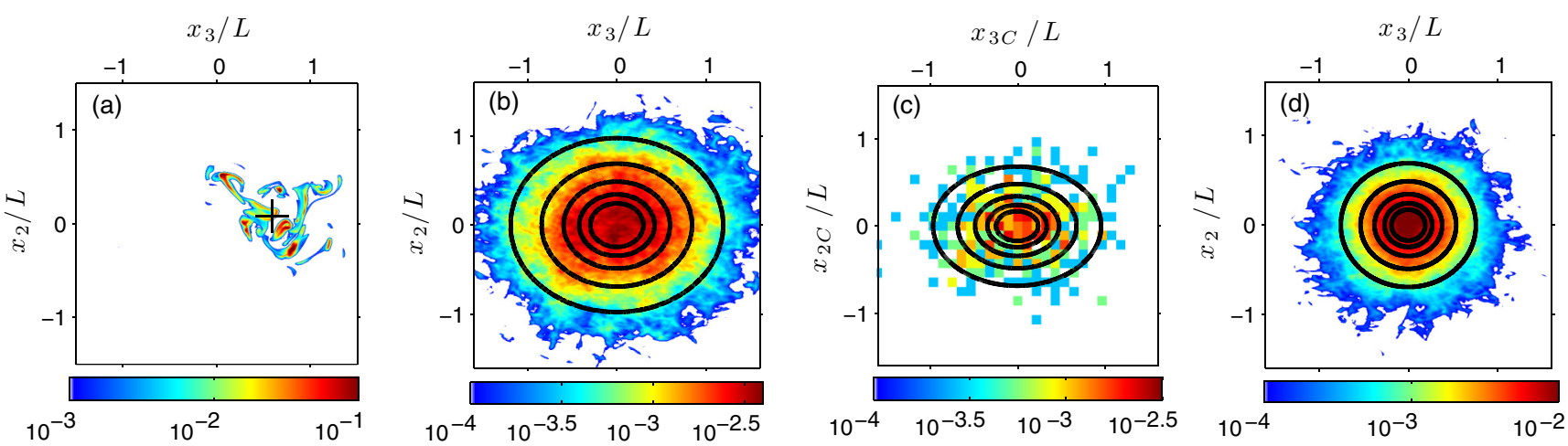

FIG. 2. (Color online) (a) A representative instantaneous concentration map with its center of mass indicated by a cross; (b) the mean concentration map in the laboratory frame; (c) the jpdf of the coordinates of the instantaneous center of mass of the plume cross section; and (d) the relative mean concentration map. All concentration values have been normalized by $C_{S}$; contours of fitted $2 \mathrm{D}$ Gaussian functions are indicated by superimposed ellipses; $x_{1} / L=20$.

two-dimensional autocorrelation maps [12]:

$$
\left\langle R\left(s_{2}, s_{3}\right)\right\rangle=\left\langle\frac{\iint C\left(x_{2}, x_{3}\right) C\left(x_{2}+s_{2}, x_{3}+s_{3}\right) d x_{2} d x_{3}}{\iint C^{2}\left(x_{2}, x_{3}\right) d x_{2} d x_{3}}\right\rangle .
$$

Representative plots of $\left\langle R\left(s_{2}, s_{3}\right)\right\rangle$ and $q\left(s_{2}\right)$ are presented in Fig. 4. The half-widths $\sigma_{Q 2}$ and $\sigma_{Q 3}$ were defined as the square roots of the second central moments of $q\left(s_{2}\right)$ and $q\left(s_{3}\right)$. The half-width ratio was $\sigma_{Q 3} / \sigma_{Q 2} \approx 1.1$, which is consistent with the expectation of isotropy in the inertial subrange. The shapes of $q\left(s_{2}\right)$ and $q\left(s_{3}\right)$ at all measurement positions could be approximated by functions of the form $e^{-s^{n}}$. As shown in Fig. 5, the value of the exponent $n$ decreased with distance from the source. At the closest measurement position $\left(x_{1} / L=5\right), n \approx 1.5$, and extrapolation of the data to the source is compatible with the near-field value $n=2$, as predicted by Batchelor. Towards the end of the measurement range, however, $n$ seemed to approach the value 0.9 , which is only slightly larger than Richardson's estimate of $n=2 / 3$. The present evolution of $n$ has a trend that is opposite to the one observed by Liao et al. [12], who found that $q(s)$ resembled Richardson's pdf near the source and approached a Gaussian

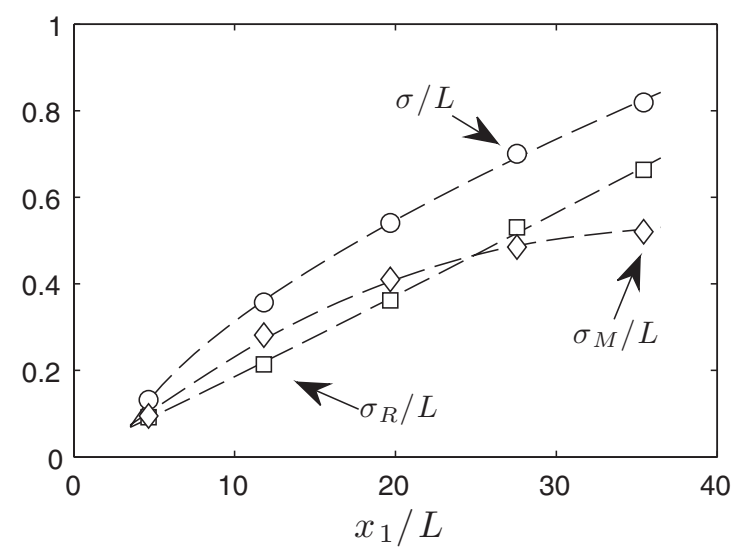

FIG. 3. (a) Evolutions of the standard deviations of the absolute mean concentration map $\sigma$, the relative mean concentration map $\sigma_{R}$, and the jpdf of the instantaneous centers of mass $\sigma_{M}$; dashed lines are included as a visual aid. shape with $n=2$ as distance from the source increased. On the other hand, the trend in Fig. 5 is in line with particle tracking results [6-8], which show that the distance-neighbor function was best described by Richardson's pdf.

The mean square particle separation $\left\langle r^{2}\right\rangle$ was estimated first as $2 \sigma_{R}^{2}$ and secondly as $\sigma_{Q}^{2}$, where $\sigma_{Q}$ is defined as the average of $\sigma_{Q 2}$ and $\sigma_{Q 3}[10,12]$. The two estimates, plotted together in Fig. 6, were nearly identical; standard deviations of the components of $\sigma_{Q}$ and $\sqrt{2} \sigma_{R}$ were on the average $\pm 6 \%$ of the mean values. The particle separations were within the inertial subrange $\left(\eta \ll \sqrt{\left\langle r^{2}\right\rangle} \ll L_{11,1}\right)$ for the entire measurement range.

The Richardson-Obukhov scaling law was fit to the measurements in the form

$$
\left\langle r^{2}\right\rangle-r_{0}^{2}=g\langle\epsilon\rangle\left(\frac{x_{1}-x_{0}}{U_{C}}\right)^{3},
$$

where $r_{0}=0.53 \mathrm{~mm}$ and the time shift $x_{0} / U_{c}$ was introduced in accordance with past practices $[7,8]$. The best-fit line, plotted in Fig. 6, was achieved for a virtual origin equal to $x_{0}=$ $-7.5 L$ and a Richardson constant of $g=0.35$; the latter value is within the range of theoretical estimates [5], although it is somewhat lower than other recent measurements [2]. For
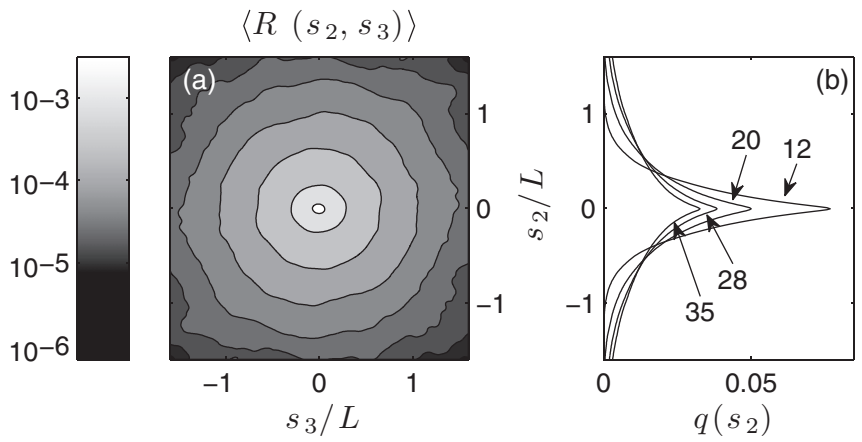

FIG. 4. (a) Map of the ensemble-averaged 2D autocorrelation $\operatorname{map}\left\langle R\left(s_{2}, s_{3}\right)\right\rangle$ at $x_{1} / L=20$ and (b) the distance-neighbor function $q\left(s_{2}\right)$ at $x_{1} / L=12,20,28$, and 35 . 


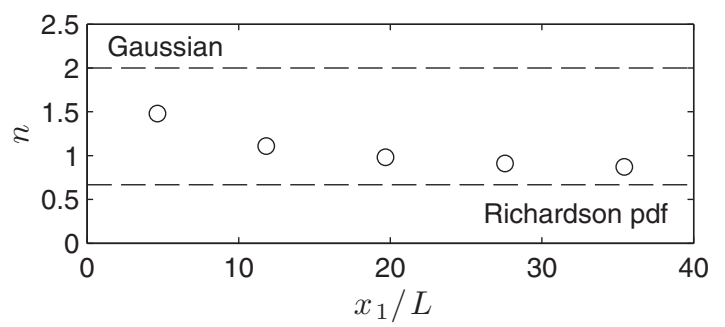

FIG. 5. Evolution of the power $n$, which describes the shape of $q(s) \propto e^{-s^{n}}$, versus streamwise distance $x_{1}$.

comparison, the Batchelor scaling law

$$
\left\langle r^{2}\right\rangle-r_{0}^{2}=\frac{11}{3} C_{2}\langle\epsilon\rangle^{2 / 3} r_{0}^{2 / 3}\left(\frac{x_{1}}{U_{C}}\right)^{2},
$$

with $C_{2}=2.13[3,4]$ and without any time shift, is also plotted in Fig. 6. It is interesting to note that this expression, which contains no adjustable parameters, was in excellent agreement with the measurements over a range that extended far beyond the Batchelor regime $\left(x_{1} / L \ll 1\right)$; although somewhat surprising, this observation is consistent with previous studies that observed $\left\langle r^{2}\right\rangle \propto t^{\gamma}$, with $\gamma$ in the range between 2 and 3 [2]. The apparent wide overlap of the Batchelor and RichardsonObukhov regimes may be attributed to the moderate Reynolds number of the present flow and hence the presence of only a narrow inertial subrange $[13,16]$, which does not permit the development of a wide and distinct Richardson-Obuhkov regime [4].

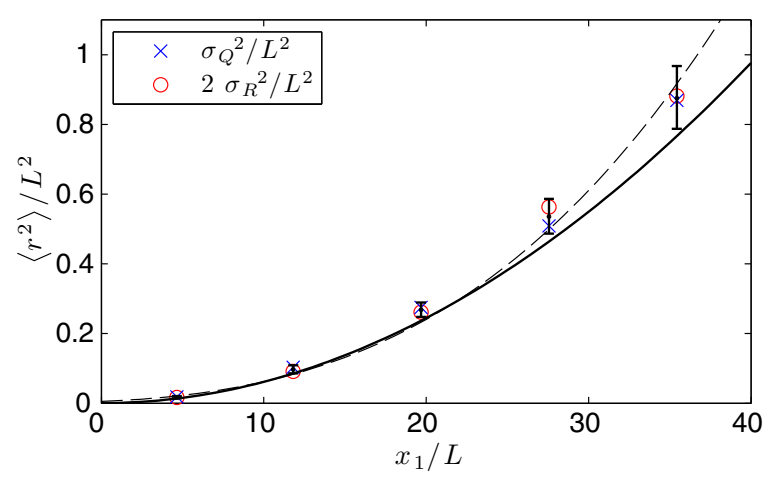

FIG. 6. (Color online) Two estimates of the streamwise evolution of the mean square particle separation; the Richardson-Obukhov scaling law is indicated by a dashed line, whereas the Batchelor scaling law is indicated by a solid line.

The main findings of this work can be summarized as follows. Estimates of particle separation in the inertial range of a scalar plume from relative mean concentration maps were essentially identical with estimates based on distance-neighbor functions. The shape of the distance-neighbor function was compatible with Richardson's expression, in line with particle tracking studies. Particle separation evolution was consistent with Richardson-Obukhov scaling with a value of Richardson's constant of $g=0.35$. The same results were also consistent with Batchelor scaling over a range that was much wider than the Batchelor regime.

Financial support was provided by the Natural Sciences and Engineering Research Council of Canada.
[1] L. F. Richardson, Proc. R. Soc. London, Ser. A 110, 709 (1926).

[2] J. P. L. C. Salazar and L. R. Collins, Annu. Rev. Fluid Mech. 41, 405 (2009).

[3] K. R. Sreenivasan, Phys. Fluids 7, 2778 (1995).

[4] M. Bourgoin, N. Ouellette, H. Xu, J. Berg, and E. Bodenschatz, Science 311, 835 (2006).

[5] B. Sawford, Annu. Rev. Fluid Mech. 33, 289 (2001).

[6] M.-C. Jullien, J. Paret, and P. Tabeling, Phys. Rev. Lett. 82, 2872 (1999).

[7] S. Ott and J. Mann, J. Fluid Mech. 422, 207 (2000).

[8] J. Berg, B. Lüthi, J. Mann, and S. Ott, Phys. Rev. E 74, 016304 (2006).

[9] G. W. Brier, J. Meteorol. 7, 283 (1950).
[10] G. K. Batchelor, Math. Proc. Cambridge 48, 345 (1952).

[11] A. S. Monin and A. M. Yaglom, Statistical Fluid Mechanics: Mechanics of Turbulence (MIT Press, Cambridge, MA, 1975), Vol. 2.

[12] Q. Liao and E. A. Cowen, J. Fluid Mech. 661, 412 (2010).

[13] C. Vanderwel and S. Tavoularis, J. Fluid Mech. 689, 434 (2011).

[14] C. Vanderwel and S. Tavoularis, in Proceedings of the Eighth International Symposium on Turbulence and Shear Flow Phenomena, Poitiers, France (unpublished).

[15] G. Csanady, Turbulent Diffusion in the Environment (D. Reidel Publishing Company, Dordrecht, The Netherlands, 1973).

[16] M. Ferchichi and S. Tavoularis, Phys. Fluids 12, 2942 (2000). 